\title{
Correction to: Painful left forearm swelling
}

\author{
Julio Brandão Guimaraes ${ }^{1,2,3} \cdot$ Renato Masson ${ }^{2} \cdot$ Marcelo Petrilli $^{4} \cdot$ Maria Teresa de Seixas Alves $^{5}$. \\ Henrique Lederman ${ }^{1,2}$
}

Published online: 7 October 2020

(C) ISS 2020

\author{
Correction to: Skeletal Radiology \\ https://doi.org/10.1007/s00256-020-03591-w
}

\section{Final diagnosis: Subperiosteal hemophilic Pseudotumor}

\section{Discussion}

Anteroposterior and lateral radiographs of the left forearm demonstrate (Fig. 1) expansile subperiosteal lytic bone lesions that extend into the soft tissue, associated with diffuse cortical thinning, subperiosteal scalloped erosion of the radius, and radiating trabeculae with periosteal reaction. Imaging findings also indicate the presence of calcifications with a curvilinear pattern in the periphery of soft tissues in the medial proximal radius and bone remodeling in the distal ulna.

The original version of this article which can be found at https://doi.org/ $10.1007 / \mathrm{s} 00256-020-03591-\mathrm{w}$ is missing figures 4,5 , and 6 . To avoid any misinterpretation, the whole article is published below in full with the corrected data.

Julio Brandão Guimaraes

Julio.BrandaoGuimaraes@ucsf.edu;

juliobrandaoguimaraes@hotmail.com

1 Department of Radiology, Grupo de Apoio ao Adolescente e à Criança com Câncer (GRAACC), Sao Paulo, Brazil

2 Department of Radiology, Federal University of Sao Paulo (UNIFESP), Sao Paulo, Brazil

3 Musculoskeletal and Quantitative Imaging Research Group (MQIR), Department of Radiology and Biomedical Imaging, University of California, San Francisco, 185 Berry St, Suite 350, San Francisco, CA 94158, USA

4 Department of Orthopedic Surgery, Grupo de Apoio ao Adolescente e à Criança com Câncer (GRAACC), Sao Paulo, Brazil

5 Department of Pathology, Federal University of Sao Paulo (UNIFESP), Sao Paulo, Brazil
MRI findings (Fig. 2) show a large encapsulated subperiosteal soft tissue mass surrounding the radius, with distinct signal intensities on both T1- and T2-weighted images, including spontaneous high signal intensity on T1, suggesting different stages of hemorrhage. Furthermore, the interosseous space is widened due to the dimension of the soft tissue mass. Postcontrast imaging did not reveal any solid areas, exhibiting a predominant peripheral enhancement distribution pattern.

MRI angiography findings (Fig. 3) showan active filling of a cystic cavity within the lesion, suggesting an intracystic hemorrhage that had been actively bleeding. The pathology of the biopsy sample confirmed the content of the pseudocyst's cavities, which were filled with hemorrhagic and fibrinoid content (Fig. 4), as well as negative for atypical cells.

Subsequently, the patient underwent surgery with curettage and iliac bone grafting, which was shortened by a massive bleeding of the donor site for the graft (Fig. 5). After a severe drop in hemoglobin, coagulation tests for factor VIII deficiency were carried out, and the patient was diagnosed with typeA hemophilia. Pathology findings, along with the newly uncovered history of type A hemophilia, are consistent with the diagnosis of a hemophilic pseudotumor. The patient showed clinical improvement after the replacement of factor VII and management of the anemia, and 6 months into recovery, there were no signs of local recurrence (Fig. 6).

Type A hemophilia is an X-linked recessive genetic disorder [1-3], and therefore, typically observed among males. The hemophilic pseudotumor, in turn, is a rare complication which affects $1-2 \%$ of patients [3-5]. The most common locations for osseous pseudotumors are the femur, pelvis, tibia, and small bones of the hand $[4,6,7]$. Similarly to recurrent bleeding into the joints, which can lead to hemophilic arthropathy, repetitive bleeding into the bones/subperiosteal region can result in osseous pseudotumors that may present on imaging studies as intraosseous and subperiosteal lesions $[1,5]$.

These lesions appear on a radiograph as well-defined, unilocular or multilocular, expansile lytic lesions, with a geographic 

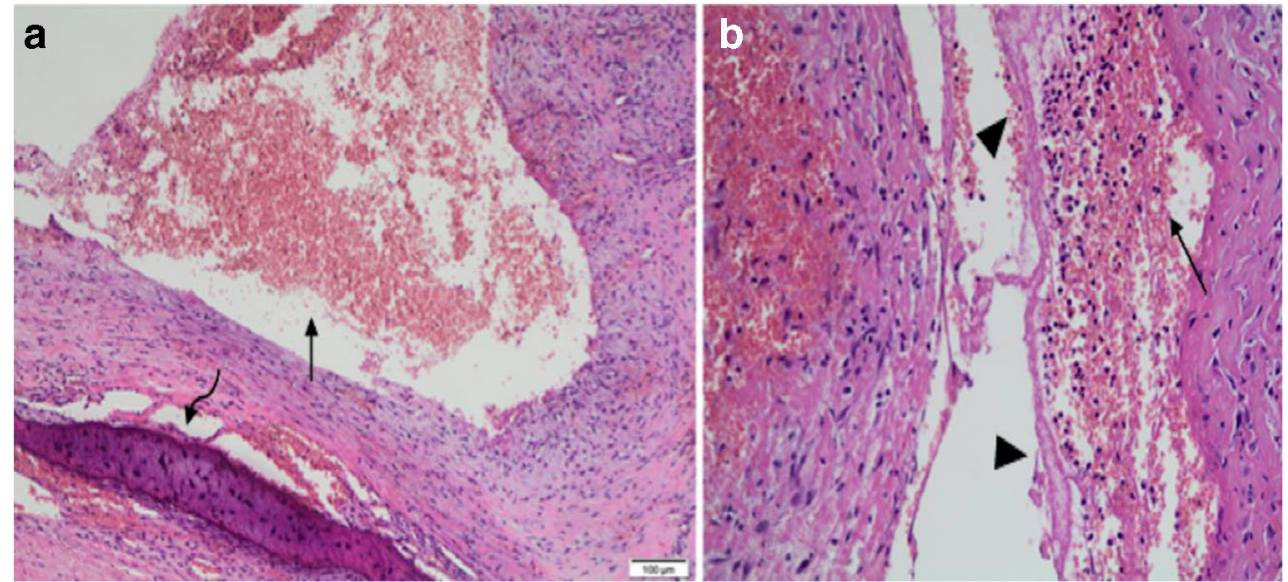

Fig. 4 Photomicrograph of the biopsy. HE 100x (a) and 400x (b) show pseudocystic cavities with hemorrhagic (arrows) and fibrinoid (arrowheads) content. Also note normal residual bone (curved arrow in a)

pattern of bone destruction. They may also co-occur with cortical thinning, peripheral sclerosis, endosteal scalloping, and dystrophic calcifications. As a matter of fact, these lesions can be extremely destructive and completely replace the involved segments of the bone. The various radiographic differential diagnosis depend on the patient's age and are related to primary and secondary bone tumors (giant cell tumor, telangiectatic osteosarcoma, plasmacytoma, metastasis, and malignant fibrous histiocytoma); tumor-like lesions (aneurysmal bone cyst, solitary bone cyst, and brown tumor); and infection [5]. MR imaging appears characteristically as multiloculated lesions containing fluid and presents different signal intensity on various sequences, as a result of the existence of blood components in several different stages of evolution. These lesions are surrounded by T1- and T2-hypointense rims, due to either hemosiderin or peripheral sclerosis/fibrous capsule, showing slight enhancement, if any, on postgadolinium images $[1,5]$.

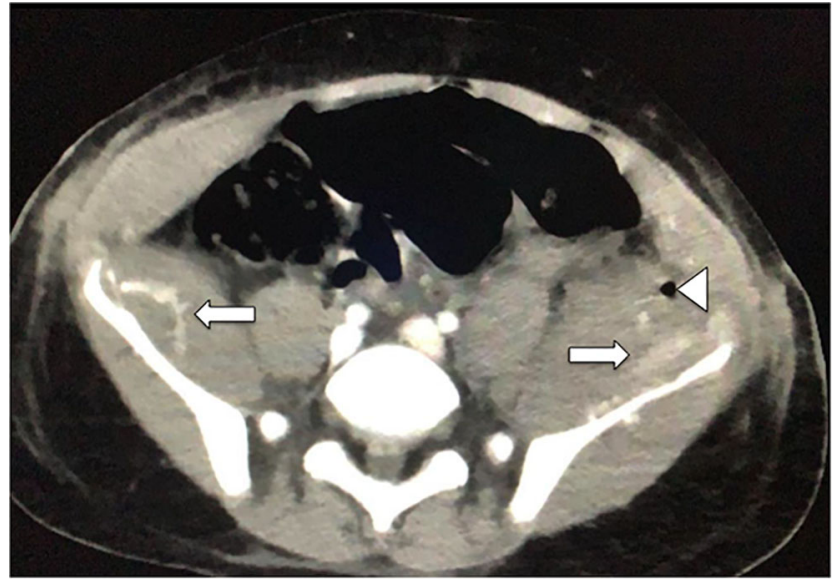

Fig. 5 Axial contrast-enhanced CT of the pelvis after bone grafting shows subperiosteal and intramuscular iliacus hematomas with active bleeding (arrows). Note small focus of gas in the left hematoma (arrowhead), secondary to surgical manipulation
Subperiosteal lesions are atypical presentations of the hemophilic pseudotumor, which result from the elevation of the periosteum caused by hemorrhage. In this scenario, radiographic findings include soft tissue masses located in the periosteum, as well as cortical erosions, subperiosteal new bone formation, and soft tissue extension. Similarly to our case, these lesions may be associated with an aggressive periosteal reaction and curvilinear calcifications at the periphery of the lesion, projecting into soft tissues, which consists in the most characteristic feature of hemophilic pseudotumors [8].

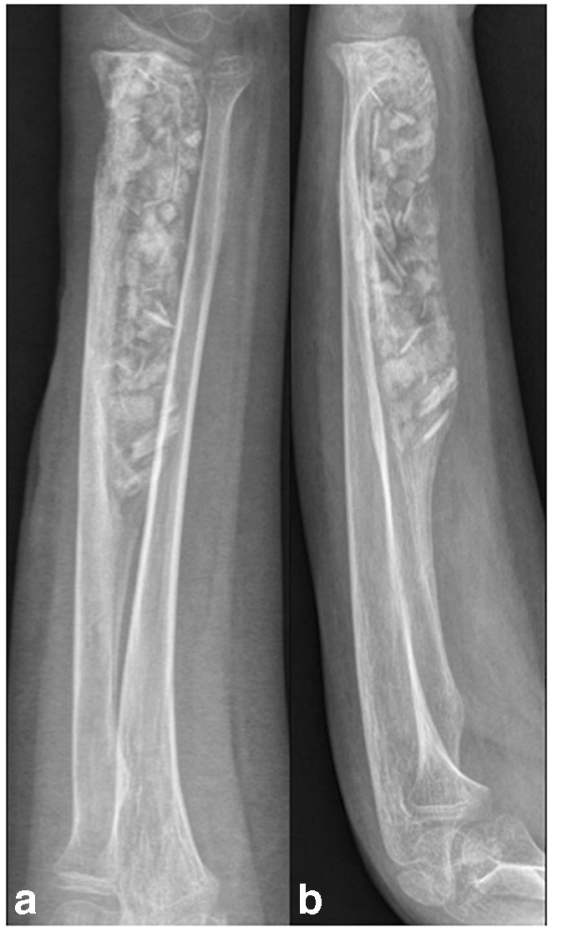

Fig. 6 Six months post-surgical CR (a and b) showing post-curettage imaging findings with bone grafting, without any signs of complications 
Hemorrhagic lesions may appear on the MRI as confined to the subperiosteal space or extended into the bone or soft tissue. Therefore, such lesions, associated with the presence of blood components in the various stages of evolution, when no solid component is observed in the peripheral capsule on the MRI, should be potentially considered subperiosteal hemophilic pseudotumors in pediatric patients, even without prior clinical history, as demonstrated in our case.

Complications from pseudotumors can be either local or systemic, including superimposed infection, fistula formation, exsanguination due to rupture, compartment syndrome, and neurovascular compression $[6,7]$.

Moreover, the management of hemophilic pseudotumors is controversial $[4,7]$. The initial management includes factor VIII replacement and control of anemia, while conservative management and watchful waiting are options for patients with minimal symptoms [4]. Although surgical treatment (drainage or excision) is very effective, it carries a high risk of complications such as bleeding, infection [6], and in extreme cases, amputation [5].

\section{Compliance with ethical standards}

Conflict of interest The authors declare that they have no conflict of interest.

\section{References}

1. Park JS, Ryu KN. Hemophilic pseudotumor involving the musculoskeletal system: spectrum of radiologic findings. AJR Am J Roentgenol. 2004;183(July):55-61.

2. Jaganathan S, Gamanagatti S, Goyal A. Musculoskeletal manifestations of hemophilia: imaging features. Curr Probl Diagn Radiol. 2011;40(5):191-7.

3. Pakala A, Thomas J, Comp P. Hemophilic pseudotumor: a case report and review of literature. Int J Clin Med. 2012;3:229-33.

4. Lim M, Nielsen Y, Ma B, Key ANS. Clinical features and management of haemophilic pseudotumours: a single US centre experience over a 30-year period. Haemophilia. 2014;20:58-62.

5. Stafford JM, James TT, Allen AM, Dixon LR. Hemophilic pseudotumor: radiologic-pathologic correlation. Radiographics. 2003;23(4):852-6.

6. Zhai J, Weng X, Zhang B, Peng H, Bian Y, Zhou L. Surgical management of hemophilic pseudotumor complicated by destructive osteoarthropathy. Blood Coagul Fibrinolysis. 2015;26:373-7.

7. Magallon M, Monteagudo J, Altisent C, Ibdiiez A, Riba J, Tusell J. Hemophilic pseudotumor: multicenter experience over a 25-year period. Am J Hematol. 1994;45:103-8.

8. Brant E, Jordan H. Radiologic aspect of hemophilic pseudotumors in bone. AJR Am J Roentgenol. 1972;115:525-39.

Publisher's note Springer Nature remains neutral with regard to jurisdictional claims in published maps and institutional affiliations. 